\title{
A NEW DESCRIPTOR FOR IMAGE RETRIEVAL USING CONTOURLET CO- OCCURRENCE
}

\author{
Nguyen Duc Hoang ${ }^{(1)}$, Le Tien Thuong ${ }^{(2)}$, Do Hong Tuan ${ }^{(2)}$, Bui Thu Cao ${ }^{(3)}$ \\ (1) Broadcast Research and Application Center, Vietnam Television (VTV-BRAC)
}

(2) University of Technology, VNU-HCM

(3) Ho Chi Minh City University of Industry

(Received December 20 $0^{\text {th }}, 2011$, Accepted March 21 $1^{s t}$, 2012)

ABSTRACT: In this paper, a new descriptor for the feature extraction of images in the image database is presented. The new descriptor called Contourlet Co-Occurrence is based on a combination of contourlet transform and Grey Level Co-occurrence Matrix (GLCM). In order to evaluate the proposed descriptor, we perform the comparative analysis of existing methods such as Contourlet [2], GLCM [14] descriptors with Contourlet Co-Occurrence descriptor for image retrieval. Experimental results demonstrate that the proposed method shows a slight improvement in the retrieval effectiveness.

Keywords: content-based image retrieval, CBIR, Contourlet Co-occurrence, Contourlet.

\section{INTRODUCTION}

Content-based image retrieval (CBIR) becomes a real demand for storage and retrieval of images in digital image libraries and other multimedia databases. CBIR is an automatic process for searching relevant images to a given query image based on the primitive low-level image features such as color, texture, shape and spatial layout [15].

In other researching trend, transformed data are used to extract some higher level features. Recently, wavelet-based methods which provide better local spatial information in transform domain have been used $[10,8,9,6$, and 7]. In [10], the variances of Daubechies wavelet coefficients in three scales were processed to construct index vectors. In SIMPLIcity [8], the image was first classified into different semantic classes using a kind of texture classification algorithm. Then, Daubechies wavelets were used to extract feature vectors. Another approach called wavelet correlogram $[9,6,7]$ used the correlogram of high frequency wavelet coefficients to construct feature vectors.

\subsection{Our Approach}

In this paper, we propose a new descriptor for image retrieval called the contourlet cooccurrence descriptor. The highlights of this descriptor are: (i) it used Contourlet transform with improved characteristics compared with wavelet transform [11, 12], (ii) it used Grey Level Co-Occurrence Matrix that considers the spatial relationship of pixels [14], (iii) the size of the feature is fairly small. Our experiments show that this new descriptor can outperform the contourlet method [2] and the GLCM method [14] using individual for image 
retrieval.

The Contourlet transform based on an efficient two-dimensional multiscale and directional filter bank that can deal effectively with images having smooth contours. The main difference between contourlets and other multiscale directional systems is that the contourlet transform allows for different and flexible number of directions at each scale, while achieving nearly critical sampling. Specifically, contourlet transform involves basis functions that are oriented at any power of two's number of directions with flexible aspect ratios [4].

The co-occurrence probabilities provide a second-order method for generating texture features [14]. These probabilities represent the conditional joint probabilities of all pair wise combinations of grey levels in the spatial window of interest given two parameters: interpixel distance $(\delta)$ and orientation $(\theta)$ [3].

The contourlet co-occurrence descriptor extract co-occurrence matrix features from subband signals of the images are decomposed using contourlet transform. First, contourlet coefficients are quantized to different levels for each subbands and scales. The quantized codebooks are generated to reduce the computation time correlation. Second, cooccurrence matrix features are calculated on interpixel distance $(\delta)$ and orientation $(\theta)$ compatible with the direction of subbands that are quantized. Finally, the extracted feature vectors are constructed from 4 common cooccurrence features.
The similarity measure using for the feature vectors that are extracted from this descriptor is also designed. Details are presented in the following sections.

\subsection{Related Works}

The computation of co-occurrence matrix features from image to describe their second order statistics is proposed in [14]. To conjecture the statistical properties with multiscale representation, the feature sets called wavelet co-occurrence signatures are introduced in [16]. Authors found that some textures are best characterized using these wavelet co-occurrence signatures.

The wavelet correlogram is other approach for image indexing / retrieval [7, 13]. According to this approach, wavelet coefficients are computed first to decompose space-frequency information of the image. These directional sub-bands enable computing the image spatial correlation in a more efficient way, while taking into consideration the semantic image information. A quantization step is then applied before computing directional autocorrelogram of the wavelet coefficients. Finally, index vectors are constructed using these wavelet correlograms [9].

Our methodology compared with wavelet correlogram have some differences in the following ways:

- Contourlet transform is used in our method. This transform allows flexible number of directions at each scale compared with wavelet transform that have only horizontal,

\section{Trang 6}


vertical, and diagonal directions.

- Co-occurrence matrices (with 6 orientations) is computed instead of wavelet correlogram (only computing on LH, HL subbands).

- Co-occurrence matrix features is extracted (their second order statistics) instead of computing autocorrelogram to construct feature vectors.

The proposed descriptor and the algorithm for CBIR were test on the database of 1000 color image including 10 different image categories. Experimental results demonstrated slightly improvement in the retrieval effectiveness of the contourlet co-occurrence method compared with the methods based on contourlet transform or GLCM.

\subsection{Overview of Paper}

Next sections of the paper are structured as follows: the Contourlet Co-occurrence descriptor and algorithm for CBIR is reviewed in Section II. Section III introduces similary measure using for the proposal algorithm. Experimental results and performance comparing with relative algorithms are given in Section IV. Finally, Section V is devoted to concluding remarks.

\section{CONTOURLET CO-OCCURRENCE}

\subsection{The Parameters for Contourlet Transform}

Contourlet transform are implemented through a double filter bank structure to decompose images on a number scales and directions. This is done by a combination of the Laplacian Pyramid decomposition with filter banks at each scale. Because of the structure of stage (level), number of direction in each level of multiscale decomposition in contourlet transform independent each other. Characteristics make contourlet transform achieve the high flexibility in image decomposition.

In our descriptor, the images are decomposed by Contourlet transform with 2 levels. The parameters as following:

- Decomposition level: [0, 2],

- Pyramidal filter: ' $p k v a$ ',

- Directional filter: ' $p k v a$ '.

Figure 1 illustrates a image is decomposed by contourlet trasform with parameters as was stated above.

In level 1, all 4 directional subbands are used in quantization step and computing cooccurrence matrix. With level 2, only horizontal and vertical subbands are used for next steps.

\subsection{Quantization of Subbands in Each Level}

Since contourlet coefficients in subbands are real numbers with a large dynamic, a quantization step is required before computed co-occurrence matrices. In each level, contourlet coefficients have the dynamic range variation. So that, 2 quantized levels (2 codebooks) is used corresponding to each level. Subband of the same level is quantized the same codebook. 


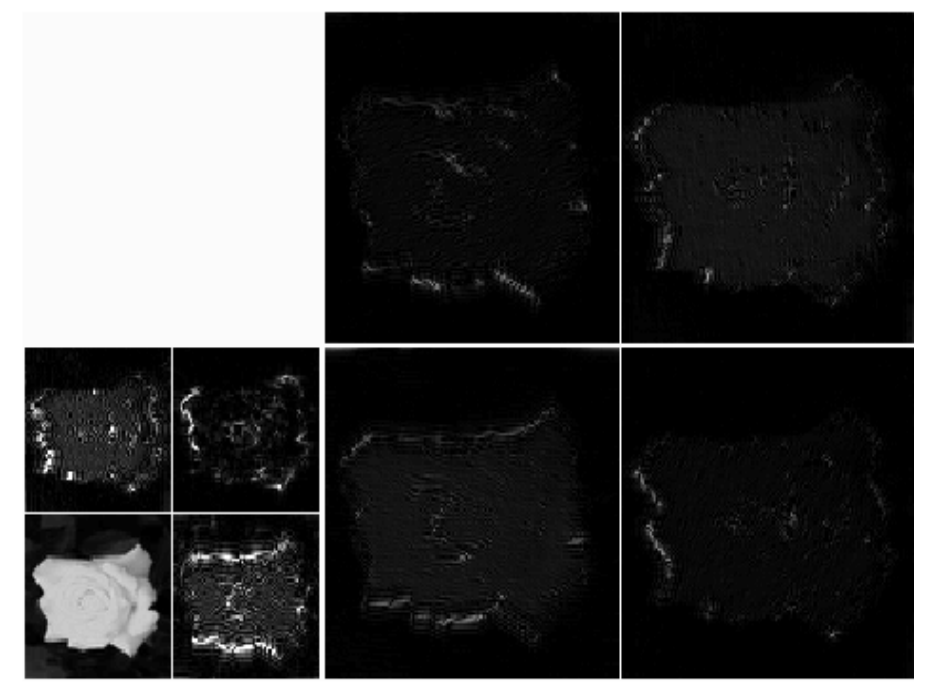

Figure 1. The illustration of contourlet transform of a image with parameters are defined.

Figure 2 shows the quantization procedure performed on 2 levels.

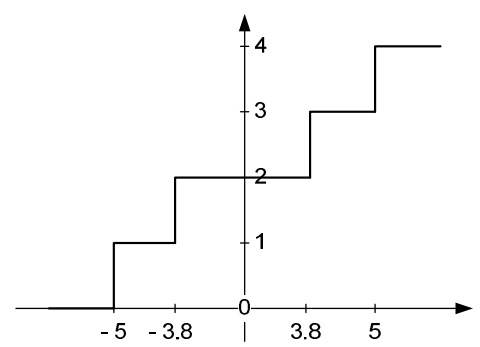

(a)

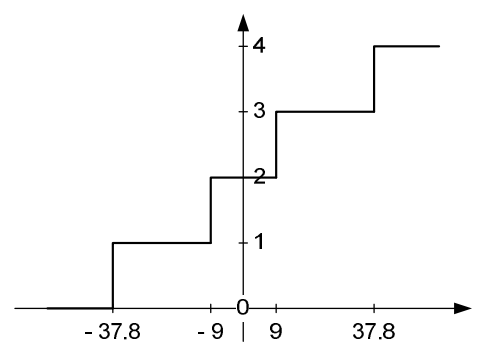

(b)

Figure 2. Quantized levels used corresponding to level 1 (a) and level 2 (b)

\subsection{Grey Level Co-occurrence Matrix} (GLCM)

The Grey Level Co-occurrence Matrix (also called the Grey Tone Spatial Dependency Matrix) is a spatial dependence matrix of relative frequencies in which two neighboring pixels that have certain grey tones and are separated by a given distance and a given angle; occur within a moving window [14].

The figure 3 illustrates the spatial relationships of pixels that are defined by this array of offsets, where $\delta$ represents the distance from the pixel of interest.

\section{Trang 8}




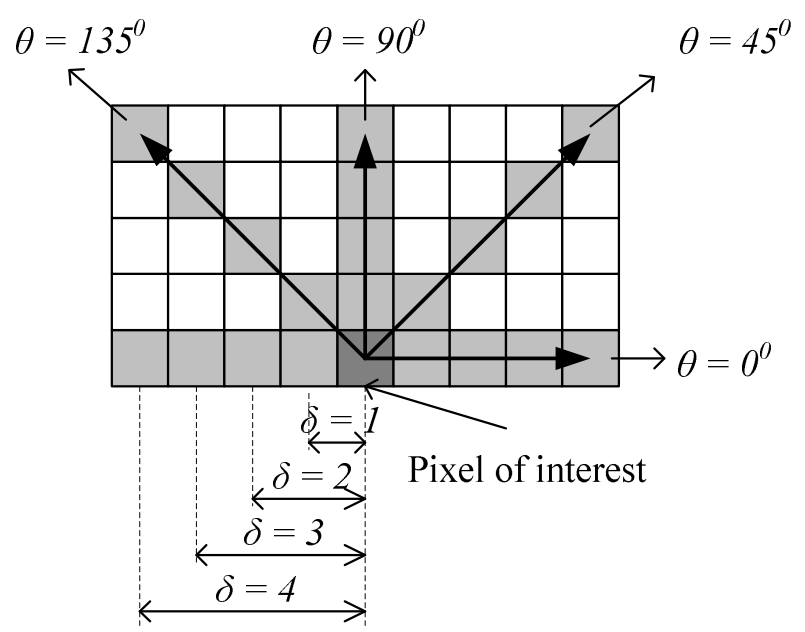

Figure 3. Four Orientations $(\theta)$ and distances $(\delta)$ in Co-occurrence matrix

The probability measure can be defined as:

$$
\operatorname{Pr}=\left\{C_{i j} \mid(\delta, \theta)\right\}
$$

where $C_{i j}$ (the co-occurrence probability between grey levels $i$ and $j$ ) is defined as:

$$
C_{i j}=\frac{P_{i j}}{\sum_{i, j=1}^{G} P_{i j}}
$$

where $P_{i j}$ represent the number of occurrences of grey levels $i$ and $j$ within the given window, given a certain $(\delta, \theta)$ pair; and
$G$ is the quantized number of grey levels. The sum in the denominator thus represent $\$ 1)^{\text {the }}$ total number of grey level pairs $(i, j)$ within window.

Although many statistics exits [14], four grey level shift invariant statistics that ${ }_{(2)}$ are commonly applied are used in our method. Statistics (Table 1) were applied to the cooccurrence probabilities to generate the features as following:

Table 1. Textural Features Extracted from GLCM

\begin{tabular}{ll}
\hline Description & Formula \\
\hline Contrast (CON) & $\sum C_{i j}(i-j)^{2}$ \\
Correlation (COR) & $\sum \frac{\left(1-\mu_{x}\right)\left(1-\mu_{y}\right) C_{i j}}{\sigma_{x} \sigma_{y}}$ \\
Uniformity (UNI) & $\sum C_{i j}^{2}$ \\
Inverse difference (INV) & $\sum \frac{C_{i j}}{1+|i-j|}$ \\
\hline
\end{tabular}


In our method, the images are decomposed

by the Contourlet transform with decomposition level: $[0,2]$. Six directional subbands are quantized and calculated GLCM as in figure 4.

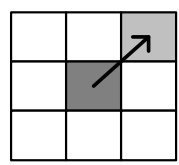

$\theta=45^{\circ}$

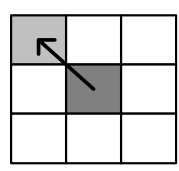

$\theta=135^{\circ}$

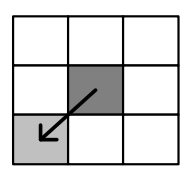

$\theta=-135^{\circ}$

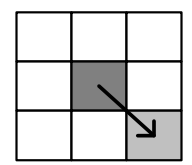

$\theta=-45^{\circ}$

(a) Decomposition level 1

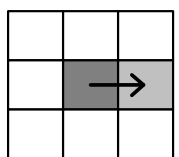

$\theta=0^{\circ}$

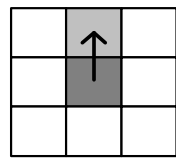

$\theta=90^{\circ}$

(b) Decomposition level 2

Figure 4. Orientations are used to calculate GLCMs for subbands

With decomposition level 1, all four directional subbands are quantized as in Fig. 2 (a) and calculated GLCMs as following:

- In first subband, the GLCMs are calculated with $\delta=\{1,2,3,4\}$ and $\theta=45^{\circ}$.

- In second subband, the GLCMs are calculated with $\delta=\{1,2,3,4\}$ and $\theta=135^{\circ}$.

- In third subband, the GLCMs are calculated with $\delta=\{1,2,3,4\}$ and $\theta=-45^{\circ}$.

- In fourth subband, the GLCMs are calculated with $\delta=\{1,2,3,4\}$ and $\theta=-135^{\circ}$.

With decomposition level 2, only horizontal and vertical subbands are quantized as in Fig. 2 (b) and calculated GLCMs as following:

- In horizontal subband, the GLCMs are calculated with $\delta=\{1,2,3,4\}$ and $\theta=0^{0}$.

- In vertical subband, the GLCMs are calculated with $\delta=\{1,2,3,4\}$ and $\theta=90^{\circ}$.
Four textural features (Table 1) is extracted from each GLCM. In each subband, GLCMs are calculated with $\delta=\{1,2,3,4\}$ and a $\theta$, total have four GLCMs. So that, feature vector structure of a gray level image is: 4 features/each GLCM x 4 GLCMs/each subband x 6 subbands/image $=96$ features that corresponding to 96 real numbers, equivalent to 384 bytes per feature vector.

In case of the color image, feature vector structure is extracted from three color components of image so the feature vector of color image is larger than three times of gray image.

\subsection{Contourlet Co-occurrence Descriptor and Algorithm for CBIR}

The Contourlet Co-occurrence descriptor extracts feature vectors in some steps as shows in the block diagram of Figure 5.

\section{Trang 10}




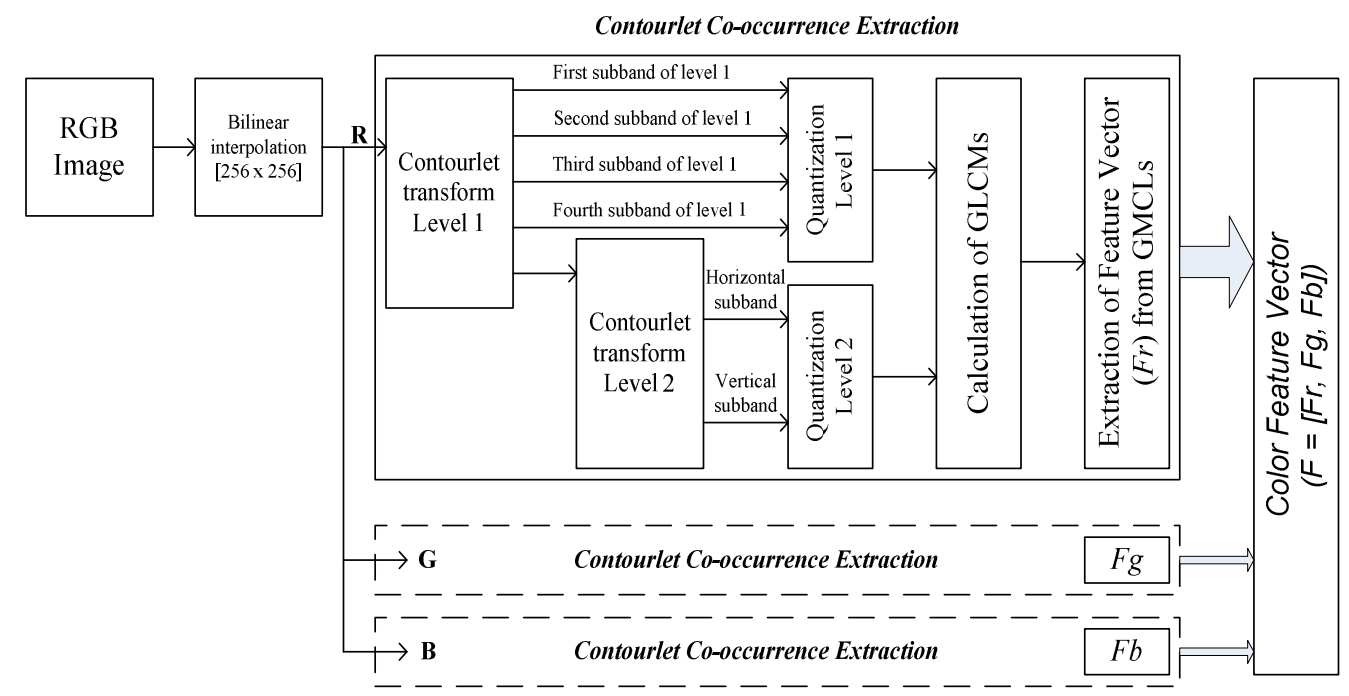

Figure 5. Contourlet Co-occurrence extraction

In this block diagram, images are without color space conversion that using $\mathrm{R}, \mathrm{G}, \mathrm{B}$ channels to calculate image features for channel separation. First, images are converted to $256 \times 256$ sizes by bilinear interpolation. This size appropriates to contourlet transform in second step. The parameters of contourlet transform are selected as above introduction. Four subbands of level 1 are quantized as in Fig 2(a). Horizontal and vertical subbands of level 2 are quantized as in Fig 2(b). Third, quantized subbands are calculated the GLCM with orientations $(\theta)$ and distances $(\delta)$ as presented in the above. Finally, the feature vectors are extracted from GLCMs on three channels.

\section{SIMILARITY MEASURES}

The image retrieval problem is following: let $D$ be an image database and $q$ be the query image. Obtain a permutation of the images in $D$ based $q$, i.e., assign rank of images in $D$ using some notion of similarity to $q$. This problem is usually solved by sorting the images $r \in D$ according to $|F(r)-F(q)|$, where $F($.$) is a$ function computing feature vectors of images and $|$.$| is some distance measure defined on$ feature vectors.

Let $F(r)=\left[f_{r j}\right]_{j=1}^{p}$ and $F(q)=\left[f_{q j}\right]_{j=1}^{p}$ be the vectors of two different images where $p$ is the feature vector length. For similarity matching, the distance measure is selected as follows:

$$
D(r, q)=\sum_{j=1}^{p} w_{j}\left|\frac{f_{r j}-f_{q j}}{1+f_{r j}+f_{q j}}\right|(3)
$$

where $w_{j}(j=1,2, \ldots, p)$ specifies the weight of each component of the feature vector.

\section{RESULTS OF WORK}

Programs are written in Matlab version R2006a and use with multiple formats of images such as GIF, JPEG, PPM, TIFF, PNG. 
The computer calculates these experiments that have the CPU speed is $2 \times 1.83 \mathrm{GHz}$ and $1 \mathrm{~GB}$ of RAM. The image database used for experiments is WANG database [5] including 1000 images that are categorized in 10 classes (including africans, beaches, buildings, buses, dinosaurs, elephants, flowers, horses, mountains, food) and each class contains 100 pictures in JPEG format.

\subsection{Search Effectiveness}

The most common evaluation measure used in Information Retrieval is recall rate and precision rate [1]. Precision rate is the probability of retrieving a image that relevant to query, and recall rate is the probability of relevant being retrieved. Let $n_{1}$ be the number of images retrieved in top 20 positions that are close to the query. Let $n_{2}$ represent the number of images in the database similar to the query. Evaluation standards recall rate and precision rate are defined as follows:

$$
\begin{aligned}
& \text { Re call rate }=\frac{n_{1}}{n_{2}} \times 100 \% \text { (4) } \\
& \text { Pr ecision rate }=\frac{n_{1}}{20} \times 100 \% \text { (5) } \\
& \text { WANG database includes } 1000 \text { natural } \\
& \text { images that are divided into } 10 \text { different } \\
& \text { categories, each including } 100 \text { images. So that, } \\
& n_{2}=100 \text {. }
\end{aligned}
$$

\subsection{Experimental Results}

To evaluate the performance of algorithm using the Contourlet Co-occurrence feature in image retrieval, two relative algorithms are used to compare with it. First algorithm is based on extracting features from coefficients in subbands of contourlet transform [2] (hereinafter call this feature is the contourlet feature). Second algorithm is based on cooccurrence signatures [14] (hereinafter call this feature is the co-occurrence feature). The computation of co-occurrence matrix features from the images use $\delta$ and $\theta$ parameters as follow: $\delta=\{1,2,3,4\}$ and $\theta=\left\{45^{\circ}, 135^{\circ},-45^{\circ}\right.$, $\left.135^{0}\right\}$.

A retrieved image will be considered a match if it belongs to the same class of the query image. Figure 6 illustrates a query result using Contourlet Co-occurrence CBIR with query image is also the image displaying on the upper left of the figure. The retrieved result include 20 images have smallest similarity measures of the flowers image category in the WANG database. Average processing time for each query in experiments is: 4.72 ( $\mathrm{sec}$ ).

Table 2 compares the performance of CBIR using the contourlet co-occurrence feature with the co-occurrence feature and the contourlet feature. In each category of the WANG database, two image queries is used to retrieval and calculate recall rate $(R)$ and precision rate $(P)$. Total averages of $R \_a v e$. and $P \_a v e$. is also calculated with the best matched retrieved images is achievable using contourlet cooccurrence method.

\section{Trang 12}


Table 2. Evaluation of CBIR Algorithms Using Contourlet Co-occurrence Feature with Co-occurrence Feature and Contourlet Feature

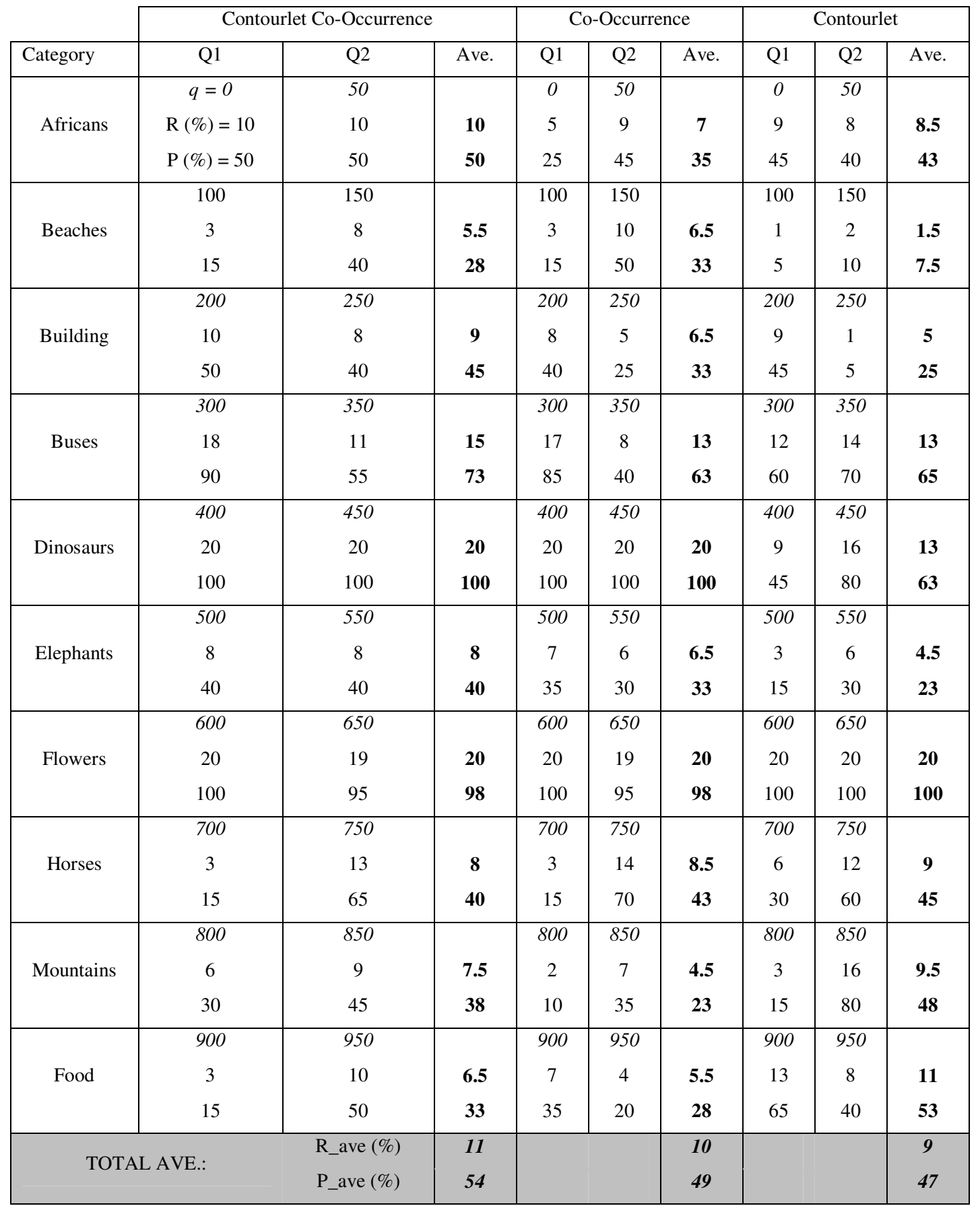




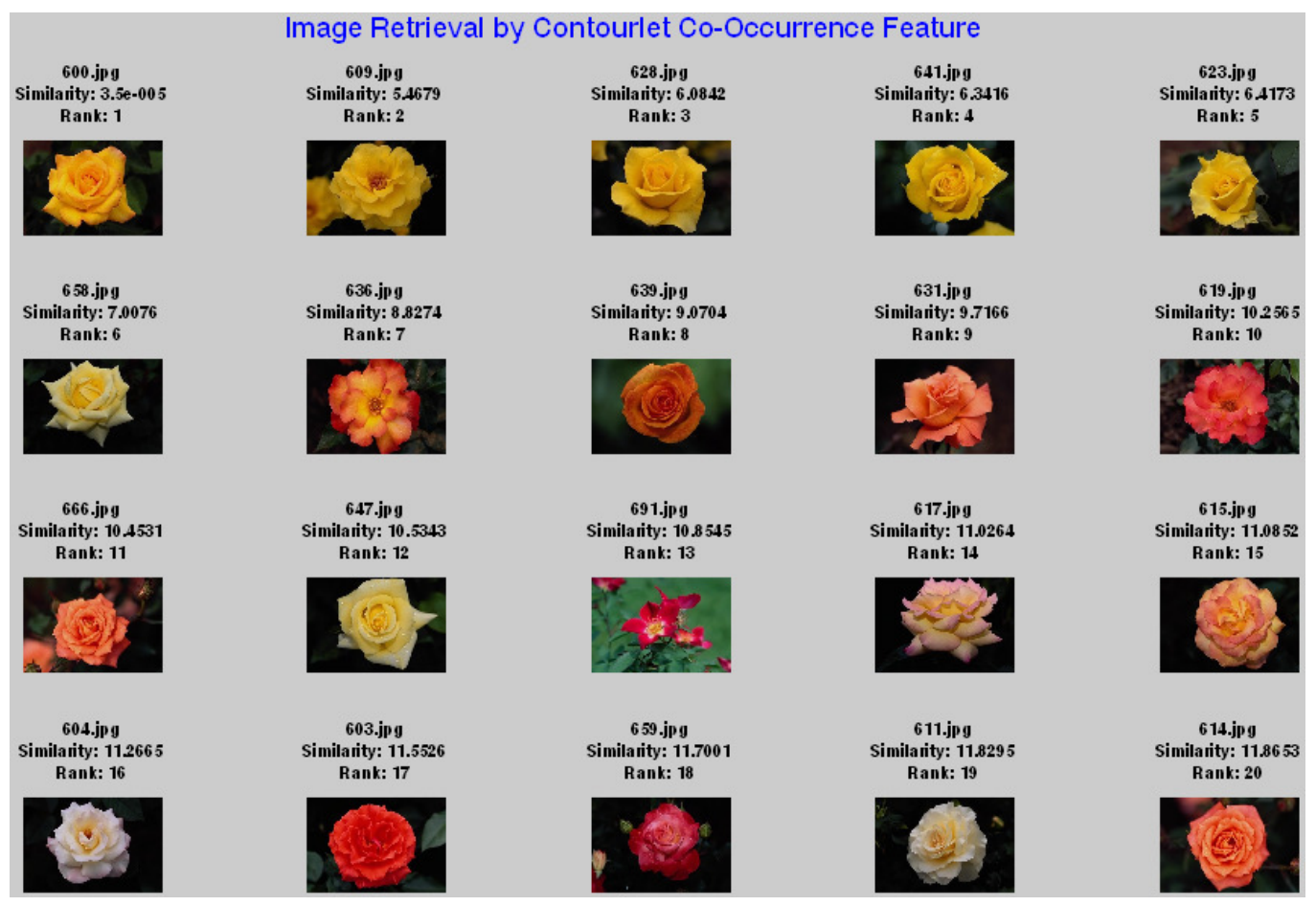

Figure 6. Results obtained from the query image 600.jpg.

\section{CONCLUSION}

In this paper, a new descriptor in CBIR was presented. Contourlet transform and GLCM matrix were combined to build a new descriptor called Contourlet Co-occurrence descriptor. The CBIR algorithm using new descriptor demonstrated higher performance compared to two relative algorithms based on contourlet feature and co-occurrence feature.

\section{Trang 14}




\title{
Bộ MÔ TẢ MỚI ÚNG DỤNG TRONG TRUY VÂN ẢNH DÙNG CONTOURLET CO- \\ OCCURRENCE
}

\author{
Nguyễn Đức Hoàng ${ }^{(1)}$, Lê Tiến Thường ${ }^{(2)}$, Đỗ Hồng Tuấn ${ }^{(2)}$, Bùi Thư Cao ${ }^{(3)}$ \\ (1) Trung tâm Nghiên cứu Ứng dụng Khoa học Kỹ thuật Truyền hình \\ (2)Trường Đại học Bách khoa, ĐGHQG-HCM \\ (3) Trường Đại học Công nghiệp Thành phố Hồ Chí Minh
}

Tóm tắt: Trong bài báo này, một bộ mô tả mới dùng để trích đặc điểm của ảnh trong các cơ sở dũ liệu ảnh được giới thiệu. Bộ mô tả mới này, gọi là Contourlet Co-Occurrence, đuợc thiết kế dụa trên sự kết hợp của biến đổi contourlet và ma trận co-occurrence mức xám (GLCM - Grey Level Co-occurrence Matrix). Để đánh giá hiệu quả của bộ mô tả đề xuất, chúng tôi thực hiện các so sánh giữa phuoong pháp dùng các bộ mô tả đặc điểm đã giới thiệu nhu Contourlet [2], GLCM [14] với bộ mô tả đặc điểm Contourlet Co-Occurrence trong ứng dụng truy vấn ảnh. Kết quả thục nghiệm đã chứng minh phuoong pháp đề xuất có hiệu quả được cải thiện hơn.

Tù khóa: CBIR, Contourlet Co-occurrence, Contourlet.

\section{REFERENCES}

[1].A.D. Bimbo, "Visual Information Retrieval", Morgan Kaufmann Publishers, Inc., San Francisco, USD, 2001.

[2]. Ch.Srinivasa Rao and S. Srinivas Kumar and B.N.Chatterji, "Content Based Image Retrieval using Contourlet Transform", ICGST International Journal on Graphics, Vision and Image Processing, GVIP, vol. 6, pp. 9-15, 2007.

[3]. Clausi, D.A., "An analysis of cooccurrence texture statistics as a function of grey level quantization", Canadian Journal of Remote Sensing, Vol. 28, No. 1, pp. 45-62, 2002.

[4]. D. D.-Y. Po and M. N. Do, “Directional multiscale modeling of images using the contourlet transform", IEEE Transactions on Image Processing, vol. 15, no. 6, pp. 1610-1620, June 2006.

[5]. http://wang.ist.psu.edu/ jwang/test1.tar [6]. H. Abrishami Moghaddam, T. Taghizadeh Khajoie, A.H. Rouhi, "A new algorithm for image indexing and retrieval using wavelet correlogram", Proc. IEEE Int'1 Conf. Image Proc., No. 2, pp 497-500, 2003.

[7]. H. Abrishami Moghaddam, T. Taghizadeh Khajoie, A. H. Rouhi, M. SaadatmandT., "Wavelet correlogram: a new approach for image indexing and retrieval", Pattern Recognition, 38(12):2506- 2518, 2005.

[8]. J. Li., J. Z. Wang, G. Wiederhold, "SIMPLIcity: emanticssensitive integrated matching for picture 
libraries", IEEE Trans. Pattern Anal. Machine Intell., 23(9):947-963, Sep. 2001.

[9]. J. Huang, S. R. Kumar, M. Mitra, W. J. Zhu, R. Zabih, "Image indexing using color correlograms", IEEE Conf. Comp. Vision and Pattern Recognition, pp. 762768, 1997.

[10]. James Z. Wang, Gio Wiederhold, Oscar Firschein and Sha Xin Wei, "ContentBased Image Indexing and Searching Using Daubechies' Wavelets", International Journal on Digital Libraries, vol. 1, no. 4, pp. 311-328, Springer-Verlag, 1998.

[11]. M. N. Do and M. Vetterli, "Contourlets", Beyond Wavelets, G. V. Welland, Ed. New York: Academic Press, 2003.

[12]. M. N. Do, "The contourlet transform: an efficient directional multiresolution image representation", IEEE Trans. Image Proc., to appear, http://www.ifp.uiuc.edu/ minhdo/public ations.

[13]. M. Saadatmand-Tarzjan, H. Abrishami Moghadam, "Enhanced wavelet correlogram methods for image indexing and retrieval", IEEE Conf. Image Processing (ICIP'06), 2005.

[14]. R. M. Haralick, Dinstein, K. Shanmugam, "Textural features for image classification", IEEE Transactions on Systems, Man, and Cybernetics, Vol. SMC-3 (November 1973), pp. 610-621.

[15]. Smeulders, A.W.M, Worring, M, Santini, S, Gupta, A, Jain, R. "Contentbased image retrieval at the end of the early years", IEEE Transactions on Pattern Analysis and Machine Intelligence, vol 22, no 12, pp.13491380. Dec 2000.

[16]. Van de Wouwer, G.; Scheunders, P.; Van Dyck, D, "Statistical texture characterization from discrete wavelet representations", Image Processing, IEEE Transactions on, Volume 8, Issue 4, pp592 - 598, Apr 1999.

\section{Trang 16}

